Cahiers $d u$ MONDE RUSSE

\section{Cahiers du monde russe}

Russie - Empire russe - Union soviétique et États indépendants

$52 / 4 \mid 2011$

Varia

\title{
Nikolaj Plotnikov, Meike Seigfried, Jens Bonnemann, éds., Zwischen den Lebenswelten
}

\section{Michail Maiatsky}

\section{OpenEdition}

\section{Journals}

Édition électronique

URL : http://journals.openedition.org/monderusse/7681

DOI : 10.4000/monderusse.7681

ISSN : $1777-5388$

\section{Éditeur}

Éditions de l'EHESS

\section{Édition imprimée}

Date de publication : 20 décembre 2011

Pagination : 842-845

ISBN : 978-2-7132-2353-2

ISSN : $1252-6576$

\section{Référence électronique}

Michail Maiatsky, « Nikolaj Plotnikov, Meike Seigfried, Jens Bonnemann, éds., Zwischen den Lebenswelten », Cahiers du monde russe [En ligne], 52/4 | 2011, mis en ligne le 03 décembre 2012, Consulté le 25 septembre 2020. URL : http://journals.openedition.org/monderusse/7681 ; DOI : https://doi.org/10.4000/monderusse.7681

Ce document a été généré automatiquement le 25 septembre 2020.

(c) École des hautes études en sciences sociales 


\title{
Nikolaj Plotnikov, Meike Seigfried, Jens Bonnemann, éds., Zwischen den Lebenswelten
}

\author{
Michail Maiatsky
}

\section{RÉFÉRENCE}

Nikolaj Plotnikov, Meike SeigrRied, Jens Bonnemann, éds., Zwischen den Lebenswelten. Interkulturelle Profile der Phänomenologie. Berlin - Münster : LIT Verlag, 2012, 261 p. (Syneidos : Deutsch-russische Studien zur Philosophie und Ideengeschichte, Bd. 3).

1 La phénoménologie constitue le point focal $\mathrm{du} 3^{\mathrm{e}}$ volume de la série des études germano-russes en philosophie et histoire des idées Syneidos intitulé Entre les mondes de la vie. Profils de la phénoménologie. Les douze contributions qui le forment sont réunies en trois « quatuors » : théorique, éthique et esthétique. Les deux premières peuvent servir de preuve de la sensibilité de la phénoménologie aux défis culturels d'aujourd'hui. L'article qui ouvre le volume, signé par Iris Därmann, témoigne du tournant produit au sein même des études phénoménologiques (et à l'instar déjà de l'ensemble des Humanwissenschaften). L'avènement du paradigme de la médialité a changé - plus tôt et plus fort qu'en France, sans parler de la Russie - la donne académique allemande, en fondant de puissantes Medienwissenschaften sur les décombres de la Germanistik jadis omnipuissante. Ce changement ne s'est pas fait sans victimes, et la première d'entre elles est, logiquement, la fameuse rigueur méthodologique. Aussi, on ne voit pas clairement si I. Därmann suggère :

- que Husserl a théorisé «involontairement » les transformations des médias de son temps, particulièrement le cinéma (le dessin animé, pourtant évoqué en guise d'appât dans le titre de l'article, ne fait qu'une apparition-éclair dans son texte); 
- qu'il a pris en compte ces transformations dans l'élaboration de la variation eidétique notamment ;

- qu'il a considéré ces médias comme une « région » parmi d'autres et méritant comme telle une application de sa méthode ;

- qu'il a simplement illustré sa méthode, forgée dans la solitude de la recherche et à l'abri des révolutions technologiques et perceptives de son temps, en tirant des exemples (certes, rares chez lui) de ces « nouveaux médias »;

- ou finalement qu'il existe de vagues « résonances » ou « similitudes » entre ces médias naissants, les recherches de Husserl, comme celles de Freud ou de Galton, les réflexions de Benjamin et jusqu'aux « vécus » d'un Hugo voyageur de train en 1837, etc.

Ce flottement n'est peut-être pas trop regrettable, puisque l'auteur réussit le principal : tracer une nouvelle perspective dans les études husserliennes, encore très cantonnées dans une lecture internaliste ${ }^{1}$.

3 L'article de Viktor Molchanov, deuxième du recueil, fait écho à l'actuel tournant «spatial » dans les sciences de l'homme. Si l'on connait les recherches précédentes de l'auteur, il est tentant de voir dans cet article une courageuse palinodie: en effet, nombre sinon la plupart des études de V. Molchanov ont été consacrées au temps et à la fameuse «conscience interne du temps» husserlienne. Ici, ce sont les dessous métaphoriques (et donc spatiaux) du temps qui sont éclairés.

Un article très fourni de Henrieke Stahl interroge l'interprétation (due à H. Dahm) de l'ouvrage majeur de Vladimir Solov'ev, Philosophie théorique, en tant que prémonition de la phénoménologie. L'auteur entreprend toute une archéologie de la controverse qui a confronté deux amis-rivaux, Solov'ev et L. Lopatin. Solov'ev n'acceptait le néocartésianisme de celui-ci que dans la perspective de la création-continuation d'une certaine mystique intellectuelle chrétienne. La religion chrétienne fut pour Solov'ev tout sauf une «région» de l'être soumise à une étude scientifique, (proto-)phénoménologique ou non.

5 La pensée encore peu connue et difficilement classable de Jósef Tischner est l'objet de la contribution de Meike Siegfried. Phénoménologue, prêtre, militant de Solidarność, Tischner fut théoricien aussi bien du théâtre que de l'existence en tant que théâtre. L'homme est un "être dramatique », et la philosophie digne de ce nom fait partie du «drame humain». La critique "scénique» de Tischner n'épargne pas la phénoménologie : celle-ci entretient avec le politique des rapports qu'elle s'est toujours refusé de thématiser, tout en partageant avec le pouvoir les velléités de "voir sans être vu, écouter sans être entendu, connaître sans être connu». Selon Tischner, le mythe platonicien de Gygès trouve un écho dans le concept husserlien du Ich transcendantal (sujet de la thèse soutenue par l'auteur en 1963 sous la direction de R. Ingarden). Ce concept serait à mettre en parallèle avec « la perspective monologique d'un spectateur solitaire et l'attitude anti-littéraire " propres à Husserl et tributaires de la primauté (et donc du pouvoir) accordée à la connaissance.

6 La dimension théâtrale relie l'œuvre de Tischner avec celle de J.-P. Sartre, analysée dans l'article de Jens Bonnemann consacré au regard et à la reconnaissance, ces deux motifs centraux de la phénoménologie sartrienne de l'intersubjectivité. Chez Sartre, comme chez bien d'autres phénoménologues (faut-il y voir le trait caractéristique de tout ce qui est de plus dynamique et fécond dans le mouvement phénoménologique ?), l'inspiration husserlienne se panache avec une critique des fondements de la doctrine de son maitre spirituel. À la thèse de la suprématie de la connaissance, Sartre a opposé 
sa propre approche: l'autre, on le rencontre, on ne le constitue pas. Pourtant, on a reproché à Sartre lui-même (p. ex. par B. Waldenfels) de pécher par " opsocentrisme ", primauté du regard. Selon Bonnemann, on aurait pu limiter, voire écarter ces reproches, si l'on avait mobilisé d'autres développements sartriens, exposés notamment dans le $1^{\mathrm{er}}$ volume de L'Idiot de la famille. Ici Sartre entreprend une autocorrection importante, en étudiant le rôle du tactile et de l'affectif dans l'éducation précoce, comme dans l'ensemble du devenir-homme.

7 Dans la perspective "nataliste », qu'elle élabore depuis un certain temps déjà, Tatiana Shchyttsova étudie, dans sa contribution au volume, "l'être-né» en tant que fondement d'une nouvelle éthique. Cette perspective semble, en effet, fructueuse, compte tenu d'un "thanatocentrisme» certain, propre à toute la tradition métaphysique, qui va du concept socratique de la philosophie en tant qu'apprentissagede-mourir jusqu'à la mort de Dieu. Ce nouveau «tournant nataliste » n'a d'autre antécédent que H. Arendt qui, dans sa Vita activa, avait rompu aussi bien avec l'obsession philosophique de la mort qu'avec «l'oubli de la naissance», et proposé de suppléer la Geburtlichkeit à la Sterblichkeit comme nouveau principe philosophique. Dans la suite de l'article, qui confronte M. Bahtin et E. Lévinas, ce moment nataliste se voit, pourtant, réduit à la présence des autres (qui sont donc une fois venus au monde) et à l'équation entre naissance et création. Mais on relèvera que dans aucune citation tirée de Bahtin le thème de la natalité n'est évoqué, et qu'aucune ligne de Lévinas n'est citée, ce qui ôte passablement au texte sa force argumentative.

8 La contribution de Guy van Kerckhoven est consacrée au visage et à la face, et combine l'approche existentialiste et celle de la théologie. Contrairement à nos habitudes de soumettre à l'analyse phénoménologique des phénomènes, l'auteur propose de considérer phénoménologiquement le concept de l'épiphanie. Il le fait en recourant aux textes de Rilke et aux études de H. Barth, H. Lipps, Hegel, Lévinas et bien d'autres. Le résultat est à la fois savant, subtil voire sublime (comme c'est souvent le cas dans ce genre de phénoménologie pieuse), et quelque peu désordonné.

9 L'article de Nikolaj Plotnikov lève le voile sur une page peu connue du mouvement phénoménologique. Les slavisants germanophones connaissent Dmitrij Čiževskij (1894-1977), historien des idées et spécialiste, entre autres, de Dostoevskij. Mais on connaît mal la jeunesse estudiantine et phénoménologique de ce savant russo-ukranien qui passa quatre semestres auprès de Husserl et Heidegger à Fribourg-en-Brisgau. Dans son projet inachevé consacré au formalisme dans l'éthique, Čiževskij renonce à établir les grands principes moraux et tente de constituer une phénoménologie d'une vie éthique, en insistant sur la pluralité de l'expérience éthique.

En ouverture de la troisième partie, Käte Meyer-Drawe esquisse une lecture phénoménologique de ce que la tradition chrétienne appelle "mandylion ", à savoir le tissu sur lequel l'image du visage du Christ s'est imprimée de façon miraculeuse, en donnant le prototype à toute icône. L'auteur inscrit cette étude dans le projet de recherche de la personne et de la subjectivité dans le transfert culturel russo-allemand. L'article de Hans Rainer Sepp touche aussi au domaine sacré, bien que dans une aire plus exotique. Il tente une description phénoménologique du dessin de l'artiste japonais du XVIII ${ }^{\mathrm{e}}$ siècle Torei Enji Bodhidharma contemplant le mur. Son but est d'exposer une interaction complexe entre se-montrer et se-retirer, et par conséquent entre le visible et l'invisible. Il étudie les diverses façons de montrer l'objet dessiné, mais aussi, ex negativo, le corps de l'artiste et celui du spectateur. 
11 Dans sa contribution, l'hispaniste franco-helvéto-belge Bénédicte Vauthier ajoute un chaînon important dans l'espace «entre les mondes de la vie» annoncé sur la couverture : en effet, la difficulté de saisir la portée théorique de Bahtin et des siens en France repose sur l'ignorance des liens qui relient ce cercle avec les théoriciens européens et surtout allemands: Dilthey, Wölfflin, Walzel, Fiedler, Hildebrand... L'auteur mène une véritable enquête non seulement sur les rapports théoriques entre les formalismes russe et européen, et entre M. Bahtin, V. Vološinov et P. Medvedev, mais aussi sur les raisons de l'oubli dont ce dernier est devenu victime en France. Des motifs stratégiques (sa critique pénétrante contre le formalisme) et des circonstances contingentes (la promotion de la mouvance formaliste en France par Jakobson Genette - Todorov) se seraient entremêlés pour arriver à ce résultat, qui commence à être modifié grâce à l'engagement de l'auteur elle-même qui, en 2008, a publié, en collaboration avec R. Comtet, une traduction de La Méthode formelle en littérature.

Ce riche recueil se clôt par un texte de cinquante pages daté 1978/1987 de Aage Hansen-Löve, éminent spécialiste du formalisme russe. Il est consacré aux contemporains des formalistes, représentants de "l'école formelle philosophique », centrée autour de l'Académie des sciences de l'art (GAHN) moscovite et de l'Institut de l'histoire des arts (GIII) pétersbourgeois. La composante phénoménologique (et en partie néokantienne) y était décisive, et son impact beaucoup plus important et conséquent que chez les formalistes. Ces dernières années, l'activité théorique du GAHN a attiré l'attention d'un nombre croissant de chercheurs. Une équipe russe et internationale dont N.Plotnikov, un des éditeurs scientifiques de ce volume, est coordinateur, a consacré plusieurs colloques et ouvrages collectifs à cette institution décimée au début des années 1930 par les répressions staliniennes. L'ouverture des archives a permis d'accéder à de nombreux documents inconnus jusqu'ici. D'autant plus louable nous paraît rétrospectivement l'intérêt que A. Hansen-Löve porta - dès les années 1970 ! - pour ce chapitre méconnu de l'histoire russe et européenne, et sa volonté de le reconstituer, en dépit du manque de sources.

\section{NOTES}

1. En outre, l'auteur n'est pas resté insensible à ce mélange d'extrême sérieux et d'humour involontaire qui est le propre de la phénoménologie. Dans une note (p.20,n.11), elle cite « une réflexion langagière rare chez Husserl »: «Le mot 'imagéité' [Bildlichkeit] n'est lui-même qu'imagé et, en tant qu'étymologie, entre ici en jeu une image qui est justement très dommageable. Aussi, je préfère... qu'il soit seulement un mot et non un concept créé à partir de son sens habituel et étymologique » (Hua XXIII, S. 324). 